\begin{tabular}{|l|l|c|l|}
\hline Eiszeitalter u. Gegenwart & 36 & $111-119$ & Hannover 1986 \\
& $2 \mathrm{Abb} ., 3$ Tab. & \\
\hline
\end{tabular}

\title{
Genese und Stratigraphie der ältesten Schotter der südlichen Iller-Lechplatte (Bayerisch-Schwaben) ${ }^{*}$ )
}

\author{
KONRAD J. RÖGNER **) \\ Lower Pleistocene (Biber glaciation), gravel, heavy minerals, size distribution, paleo-icelobe margins \\ German Alpine Foreland (Iller-Lech area), Bavaria \\ TK 26: Nr. 7929, 7930, 8028
}

Kurzfassung: Die ältesten Schotter der südlichen IllerLechplatte (Hochfirst, Stoffersberg, Plattenberg-Arlesrieder) haben pleistozänes Alter. Während ihrer Akkumulationsphase haben Ur-Iller und Ur-Lech über das gleiche Einzugsgebiet verfügt wie die Gletscher, deren umgelagerte Sedimente die donaueiszeitlichen und jüngeren Schotterkörper aufbauen.

Daher ist anzunehmen, daß auch schon während der ältestpleistozänen Biberkaltzeiten im inneralpinen Raum Vereisungen existiert haben. Die Gletscher müssen dabei den heutigen morphologischen Alpenrand erreicht haben. Eine exakte Angabe der ehemaligen Eisrandlagen aus den Bibereiszeiten ist nicht möglich. Sie wären weit südlich der jüngeren donau-, günz-, mindel-, riß- und würmeiszeitlichen Moränen zu suchen und sind der glazialen, fluvioglazialen sowie periglazialen Erosion zum Opfer gefallen.

[Genesis and Stratigraphy of the oldest Gravel Bodies in the Southern Iller-Lech Area (German Alpine Foreland)]

Abstract: The oldest gravel bodies in the Southern IllerLechplatte area (German alpine foreland) as the Hochfirst and the Stoffersberg are surely of pleistocene age. The palaeo-rivers of the Iller and the Lech must have had during the accumulation the same catchment area as the glaciers and the fluvioglacial rivers of the Donau- and the following younger glaciations. Therefore it is to assume, that during the oldest-pleistocene Biber-cold-epoch glaciations in the inneralpine area have existed too. The glaciers advanced the morphological border of the Northen Alps. It's impossible to localize the palaeo- ice lobe margins. They should be searched a lot of kilometers southern to the morains of the Donau-, Günz-, Mindel-, Riß- and Würm-glaciations and they have been destroyed by the following glacial, fluvioglacial and periglacial erosion processes.

*) Herrn Prof. Dr. Hans Graul zum 75. Geburtstag.

**) Anschrift des Verfassers: Dr. K. RÖGNER M.A., Universität-Gesamthochschule Paderborn, Fach Geographie, Warburger Straße 100, 4790 Paderborn.

\section{Einleitung}

Auf der südlichen Iller-Lechplatte dominieren im Gegensatz zum nördlichen Bereich, der überwiegend von ältestpleistozänen Schottern aufgebaut wird (LÖSCHER 1976), Akkumulationen aus dem Jung-, Mittel- und Altpleistozän. ${ }^{1}$ ) Die ältestpleistozänen Ablagerungen, die hier einmal vorhanden waren, sind von den Schmelzwasserströmen der jüngeren Vereisungen in starkem Umfange erodiert worden, sodaß sie heute nur noch kleinflächig in Erscheinung treten. Dieser Eindruck entsteht zumindest dann, wenn man die neueren geologischen Karten (STREIT et al. 1975; SCHOIZ 1983) betrachtet. Die Verbreitung der ältestpleistozänen Schotter würde sich noch weiter reduzieren, wenn man Teile von ihnen ins Pliozän stellen würde, wie es schon von EBERL (1930) vorgeschlagen und neuerdings wieder von MADER (1976) gefordert worden ist. $\mathrm{Da}$ in letzter Zeit verschiedentlich die Frage gestellt wurde, ob nicht doch die ältesten Schotter der Iller-Lechplatte ins Pliozän zu datieren seien, möge diese kleine Abhandlung unter anderem der Beantwortung dieser Frage dienen.

1) Die vorliegende Pleistozängliederung geht auf GRAUL (1962: 270) zurück. Sie wurde leicht abgeändert und erweitert und ist fast identisch mit der Gliederung von JERZ \& GROTTENTHALER (1981).

Zwischen Günz- und Donauvereisung käme die BRUNHES-/ Matuyama-Grenze zu liegen, der Beginn des Pleistozäns wäre vor 2,3 bis 2,5 Millionen Jahren anzusetzen.

Jungpleistozän: Würmeiszeit und R/W-Interglazial Mittelpleistozän: Rißeiszeit und M/R-Interglazial Altpleistozän: Mindel- und Günzeiszeit sowie das G/M und Teile des D/G-Interglazials

Ältestpleistozän: Donaueiszeiten, Biberkalt(eis?)zeiten nebst den zwischenliegenden wärmeren Zeitabschnitten. 
Grundlagen der folgenden Ausführungen sind $\mathrm{Ge}$ ländearbeiten der letzten zehn Jahre, die zuerst im Südosten (Rothwaldfeld), dann im östlichen Mittelteil (Staudenplatte) und neuerdings im Südwesten der Iller-Lechplatte durchgeführt wurden.

\section{Forschungsgeschichte}

Für A. PENCK ${ }^{2}$ ) waren alle extramoränalen Schottervorkommen der Iller-Lechplatte eiszeitliche, fluvioglaziale Ablagerungen der vier klassischen Eiszeiten (Würm, Riß, Mindel, Günz). Da sie im, schwäbischen Typ' der Schotterterrassentreppe abgelagert worden waren, wobei die ältesten Schotter die am höchsten gelegenen waren und die jüngsten die Talböden bildeten, wurden die hypsometrisch über den älteren Deckenschottern gelegenen Schotterreste wie der Staufersberg als tektonisch verstellte (= herausgehobene) altpleistozäne Sedimente angesprochen (PENCK \& BRÜCKNER 1901-1909: 45; Fig. 6 mit dem Staufersberg).

EBERL vermutete in den Ottobeurer Schottern und auch im Hochfirst Reste der ,seit langem vermißten pliozänen Schotterströme" (1930: 315).

Für SCHAEFER stammen die Ottobeurer Schotter aus dem Oberpliozän und ,können durchaus schon klimatisch (kaltzeitlich) bedingt gewesen sein" (1951: 112). Der Schotter auf dem Hochfirst gehört nach Angaben des gleichen Autors zu seiner ,prädonauzeitlichen, ältestpleistozänen Gruppe (Höhenterrassen)" (SCHAEFER 1953: 47); diese Gruppe nannte er später Biberkaltzeiten (SCHAEFER 1965).

SinN faßte den Hochfirstschotter zusammen mit dem Markt Walder als eine Ablagerung der älteren Donaueiszeit (1972: 43) auf, der Plattenberg-Arlesrieder sei allerdings eine Akkumulation der jüngeren Donaueiszeit. Die Ottobeurer Schotter sind für SINN (1972) keinesfalls pliozäne Sedimente, sie bilden vielmehr die periglazial-fluviatile Liegendfazies der ältest- und altpleistozänen Schotter.

JERZ et al. (1975) konnten weitgehend die stratigraphischen Ergebnisse SinNs bestätigen, und da die geologische Karte 1:200000 Blatt CC 8726 Kempten (Bearbeitung des Quartärs durch H. ScHOLZ) sich für die Iller-Mindel-Region auf STREIT et al. (1975) ${ }^{3}$ ) bezieht, ist die dort zur Anwendung gekommene

2) In dem gemeinsam mit E. BRÜCKNER verfaßten Werk „Die Alpen im Eiszeitalter" hat PENCK zusammen mit seinem Assistenten A. E. FORSTER die grundlegenden Ergebnisse auf der Iller-Lechplatte erarbeitet.

3) Dem Aufsatz JeRz et al. (1975) ist die geologische Karte 1:100000 (Geologische Karte des Iller-Mindel-Gebietes) beigegeben, die von STREIT et al. (1975) bearbeitet wurde. stratigraphische Konzeption mit der von JERZ et al. (1975) identisch.

Auch bei der geologischen Kartierung des Meßtischblattes Amendingen durch JERZ (1978) entspricht die Stratigraphie der von 1975.

Die Tatsache, daß die bisher vorliegenden eigenen Ergebnisse auf der südwestlichen Iller-Lechplatte erhebliche Differenzen ${ }^{4}$ ) zum bisher publizierten Material aufweisen, ist ein weiterer Grund für die folgende kleine Arbeit.

\section{Ergebnisse}

Neben Hochfirst (Blatt Markt Rettenbach) und Stoffersberg (Blatt Buchloe) war vor allem die alte Kiesgrube am Schützenhaus von Daxberg (Blatt Amendingen) Schlüsselstelle für das Verständnis für die ältest-pleistozänen Schotter. Ohne die zumindest übersichtsartige Kenntnis der tertiären Schwemmfächer am Alpennordrand (Hochgrat- und Nesselburgfächer) wären folgende Aussagen nicht zu treffen.

3.1. Der Schotterrest auf dem Hochfirst (Abb. 1; = Westlicher Hochfirst der TK 8028; Rechtswert: 04 400, Hochwert: 18650) ist der älteste Schotter auf Blatt Markt Rettenbach. Von dieser ältesten Akkumulation ist nur noch ein kleiner Rest vorhanden, der an der Nordspitze der Schotterkuppe in $697 \mathrm{~m}$ Höhe der tertiären Oberen Süßwassermolasse auflagert.

Der Hochfirstschotter kann, und das ist neu, zusammen mit dem Plattenberg-Arlesrieder Schotter (SINN nahm 1972 eine Akkumulation in zwei verschiedenen Rinnen unterschiedlichen Alters an) als eine einheitliche Ablagerung angesehen werden, denn weder die von SINN (1972: 43; $702 \mathrm{~m})$ noch die von SCHAEFER (1951: 110; 691/94 m) angegebenen Höhenwerte für die Auflagerungsbasis stimmen.

Die Höhe des Hochfirstes beträgt nämlich nicht, wie in der Karte angegeben, $709 \mathrm{~m}$, sondern nur $703 \mathrm{~m}$. Mehrfach durchgeführte Höheneinmessungen mit bis zu drei barometrischen Altimetern gleichzeitig, ausgehend von verschiedenen Höhenfixpunkten (und nicht von Höhenkoten in der Karte), lieferten das gleiche Ergebnis von $703 \mathrm{~m}$.

$\mathrm{Da}$ der Schotter noch $6 \mathrm{~m}$ mächtig ist, liegt seine Basis bei den genannten $697 \mathrm{~m}$. Das sind $17 \mathrm{~m}$ über der Basis des Plattenberg-Arlesrieder Schotters bei Rufen auf eine Entfernung von knapp $2,5 \mathrm{~km}$. Das Gefälle zwischen Hochfirst und Rufen ist mit 6,8\%

4) So ist auf den genannten kleinmaßstäblichen Karten die Verbreitung der Mindelschotter und -moränen in der Gegend von Markt Rettenbach-Ronsberg zu großzügig ausgefallen. Nachweislich ältere Schotter, wie der von Speckreu werden deshalb nicht ausgeschieden. 


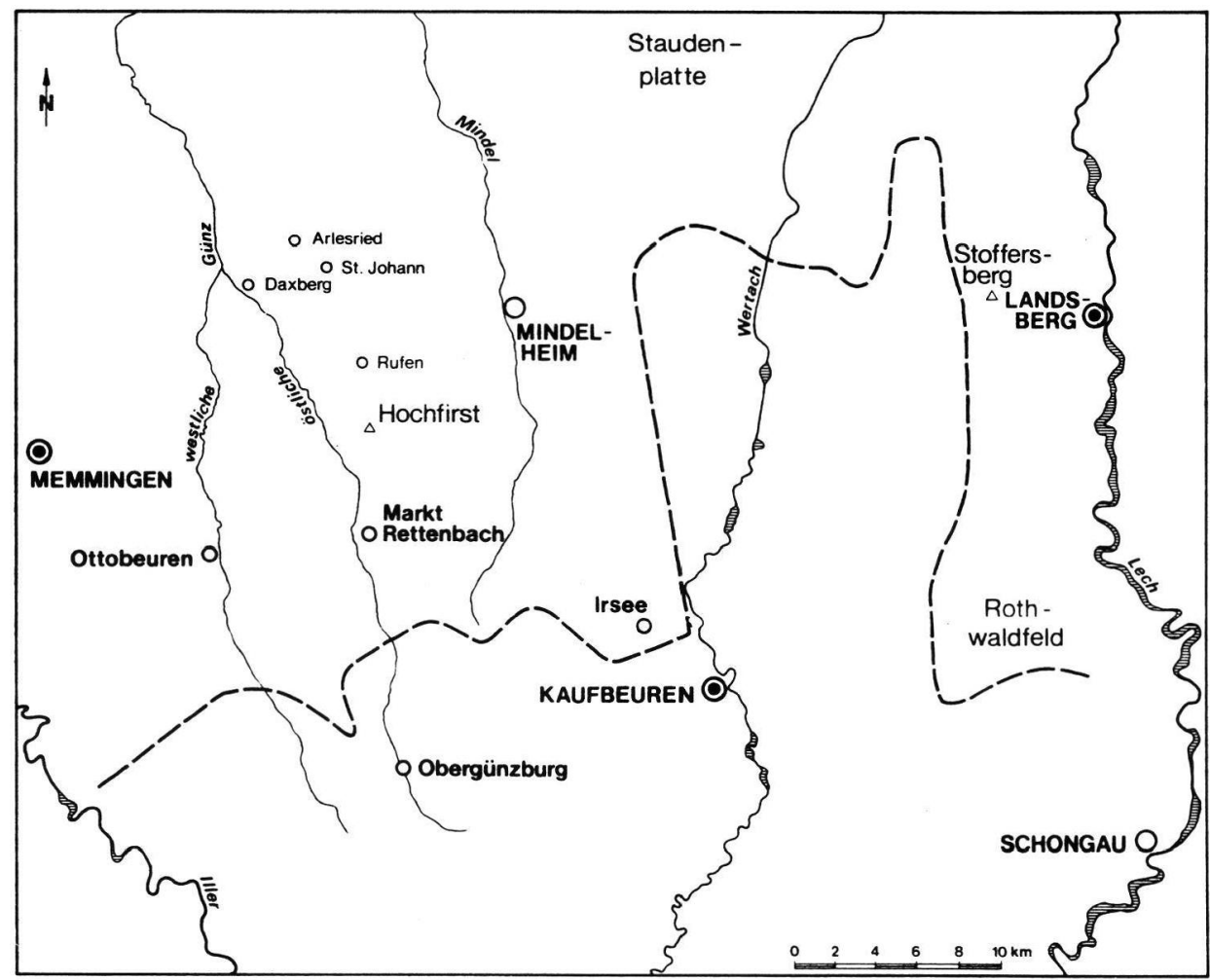

Abb. 1: Übersichtskarte der südlichen Iller-Lechplatte mit Angabe verschiedener genannter Orte. Die gestrichelte Linie gibt die ungefähre Nordgrenze der Altmoränenverbreitung an.

Tab. 1: Petrographische Analysen verschiedener ältestpleistozäner Schotter (Fraktion 2-6,3 cm $\varnothing$ )

Hochfirst

Daxberg
hangend

\begin{tabular}{lccccc}
\hline Gelbe Kalke: & $4,7 \%$ & $7,3 \%$ & $\left.14,1 \%^{3}\right)$ & $2,4 \%$ & $20,5 \%$ \\
Wettersteinkalke: & $1,1 \%$ & $0,8 \%$ & 0 & 0 & $2,1 \%$ \\
Rote Kalke: & 0 & $4,0 \%$ & 0 & 0 & $2,3 \%$ \\
Übrige Kalke: & $88,8 \%$ & $77,2 \%$ & 0 & $83,7 \%$ & $68,0 \%$ \\
\hline Kalke gesamt: & $94,6 \%$ & $89,3 \%$ & $14,1 \%$ & $86,1 \%$ & $92,9 \%$ \\
Kristallin: & $1,8 \%$ & $1,7 \%$ & $8,1 \%$ & $7,1 \%$ & $1,0 \%$ \\
Hornsteine: & $2,4 \%$ & $3,3 \%$ & $35,7 \%$ & 0 & $2,9 \%$ \\
Quarz/Quarzite: & 0 & $0,8 \%$ & $29,7 \%$ & $0,7 \%$ & $1,1 \%$ \\
Sandsteine: & $1,2 \%$ & $4,9 \%$ & $12,4 \%$ & $6,0 \%$ & $2,1 \%$ \\
Dolomite: & $\left.30-40 \%{ }^{1}\right)$ & $\left.25,1 \%{ }^{2}\right)$ & $\left.0^{4}\right)$ & 04 & $15,3 \% 5)$ \\
\hline
\end{tabular}

Anmerkungen: 1) Bis auf ca. $3 \%$ waren alle Dolomitgerölle verascht, der Wert 30-40\% ist geschätzt. 2) Der tatsächlich vorhandene Gehalt lag ursprünglich höher. Eine Schätzung erlaubt einen Wert von mindestens $30-40 \%$ anzunehmen. 3) Die gelben Kalke liegen meist als Kieselskelette vor, aus denen das Karbonat entfernt wurde. 4) Dolomite fehlen in den Analysen, sie treten ganz vereinzelt im liegenden Schotter auf. 5) Ursprünglicher Dolomitgehalt wird auf weit über $30 \%$ geschätzt. Die Schätzungen gehen auf die im Schotter vorhandenen Dolomitaschen zurück. 
zwar etwas höher als mit 5,5\% zwischen letztem Ort und St. Johann, ein Altersunterschied ist daraus nicht abzuleiten.

Die petrographische Zusammensetzung des Schotters stellt ihn eindeutig in die Reihe der quartären Ablagerungen (Tab. 1). Ein Vergleich des Hochfirstschotters mit dem fluvioglazialen, hangenden Sediment in der Kiesgrube am Schützenhaus von Daxberg erlaubt, den Hochfirstschotter als Akkumulation eines fluvioglazialen Schmelzwasserflusses anzusehen, denn er unterscheidet sich in weit deutlicherem Maße von den periglazialen $(=$ mittlerer und liegender Teil der Kiesgrube Daxberg) als von den fluvioglazialen Schottern.

Allein die Tatsache, daß der Hochfirstschotter fluvioglazialen Sedimenten ähnelt, stellt ihn nicht ins Pleistozän. Da aber in unseren Breiten die Klimaverschlechterung, welche zum Auftreten der Vereisungen führte, erst im Pleistozän eingesetzt hat, können Schotter mit fluvioglazialem Charakter nur im Pleistozän abgelagert worden sein.

Das Geröllspektrum des Hochfirstschotters weist auf ein Herkunftsgebiet in den nördlichen Kalkalpen hin. So machen Kalke auch unter Berücksichtigung der $30-40 \%$ veraschten Dolomite mehr als die Hälfte der Gerölle aus, der Kristallingehalt ist mit 1,7\% niedrig. Die reichlich vorkommenden Dolomite lassen nicht zu, den Hochfirstschotter allein auf periglazialfluviatil umgelagertes Adelegg-Material zurückzuführen. Das Liefergebiet für den Hochfirstschotter muß zur Zeit seiner Ablagerung ähnlich dem Einzugsgebiet gewesen sein, dessen umgelagerte Sedimente das Hangende der Kiesgrube Daxberg bilden.

3.2. Der Stoffersberg (Abb. $1 ;=$ Oberer Riedberg der TK 7930; R $11000, \mathrm{H} 24470$ ) ist die älteste Akkumulation im östlichen Lechgletschervorland. $\mathrm{Da}$ eine ausführliche Beschreibung bereits vorliegt (RÖGNER 1979: 81-84), brauchen hier keine weiteren Angaben zu erfolgen. Auf der südlichen IllerLechplatte fehlen zwischen Hochfirst und Stoffersberg vergleichbare ältestpleistozäne, bibereiszeitliche Sedimente. Sie treten erst wieder weiter im N zum Beispiel in der Staudenplatte südwestlich von Augsburg auf.

\subsection{Die Schotter der alten Kiesgrube am Schützenhaus von Daxberg}

(Abb. 1; TK 7929, R 98 290, H 25 590)

\subsubsection{Geländebefund und petrographische Analysen}

Die erste Beschreibung und Dokumentation der verschiedenen Schotterfazies von Daxberg erfolgte durch SINN (1972: 50ff.). Neben einer dolomitreichen, kristallinarmen hangenden Nagelfluh konnte SINN im Liegenden einen dolomitarmen, kristallinreichen Schotter nachweisen, der selbst wiederum unterteilt ist (1972, Fig. 6 und Tab. 5). Das Alter des fluvioglazialen Hangendschotters ist nach SINN günzeiszeitlich, das der liegenden periglazialen Ablagerungen ebenfalls.

JERZ (1978) fand weit bessere Aufschlußverhältnisse anläßlich des Schützenhausbaus vor. Er konnte neben einer Bestätigung der SINN'schen Ergebnisse („Günz im klassischen Sinne") vor allem die Aufschlußverhältnisse photographisch dokumentieren (JERZ 1978, Abb. 11) $)^{5}$.

Auch ich hatte die Möglichkeit, die gut aufgeschlossene Kiesgrube während der Baumaßnahmen in den Jahren 1976 und 1977 mehrfach aufzusuchen, sie zu dokumentieren und Probenmaterial zu sammeln. Die eigenen Ergebnisse sind nur teilweise eine Ergänzung zu SINN und JERZ, sie sind vor allem eine Hilfe bei der Bewertung der ältesten Schotter der südlichen Iller-Lechplatte.

Nach den in Tabelle 1 vorgestellten petrographischen Schotteranalysen ist von drei eindeutig von einander zu trennenden Sedimentationskörpern auszugehen, deren Abfolge der Abbildung 2 zu entnehmen ist.

Der hangende Bereich (zwischen 643/44 m und der Geländeoberfläche in $652 \mathrm{~m}$ ) wird von einer dolomitreichen, kristallinarmen, groben Schotternagelfluh eingenommen, auf der sich in den obersten 2 bis $5 \mathrm{~m}$ ein Boden ausgebildet hat. Nach unten wird diese fluvioglaziale Akkumulation vorwiegend durch ein Sandband abgeschlossen, stellenweise tritt in gleicher stratigraphischer Position auch ein tonig-schluffiger Horizont $^{6}$ ) auf. Da beide Fazies auch weiter unterhalb vorkommen, zählen sie zum mittleren Schichtpaket, einem feinkörnigen, ,hartereichen', dolomitfreien Schotter.

Diese mittlere Akkumulation mit sehr vielen Quarzen, Quarziten und Hornsteinen, aber auch mit vielen Sandsteinen und Kristallingeröllen, ist verschiedentlich durch Eisen- und Manganoxidbänder rötlich oder schwärzlich gefärbt. Der Schotter, der in der vorgestellten Analyse (Tab. 1) noch $14 \% \mathrm{Kalke}^{7}$ ) enthält, ist an anderer Stelle vollständig kalkfrei. Der Grad der

5) Das ist vor allem vor dem Hintergrund interessant, daß die Wand inzwischen wieder verwachsen ist und infolge der Baumaßnahmen nicht mehr zu begehen ist. 6) Trotz intensivster Suche konnten in diesen entweder primär kalkfreien oder nachträglich entkalkten verschiedenen Horizonten keine Konchylien gefunden werden.

7) Und diese Kalke sind meist nur noch als Kalk- oder Kieselskelette erhalten. Der geringe Kalkanteil wird durch Ananlysen untermauert, die für die Matrix (Fein- und Mittelsand, Schluff und Ton) einen Karbonatgehalt von etwa $5 \%$ ausweisen, im hangenden Schotter wurden über $50 \%$ ermittelt. 
Geländebefund

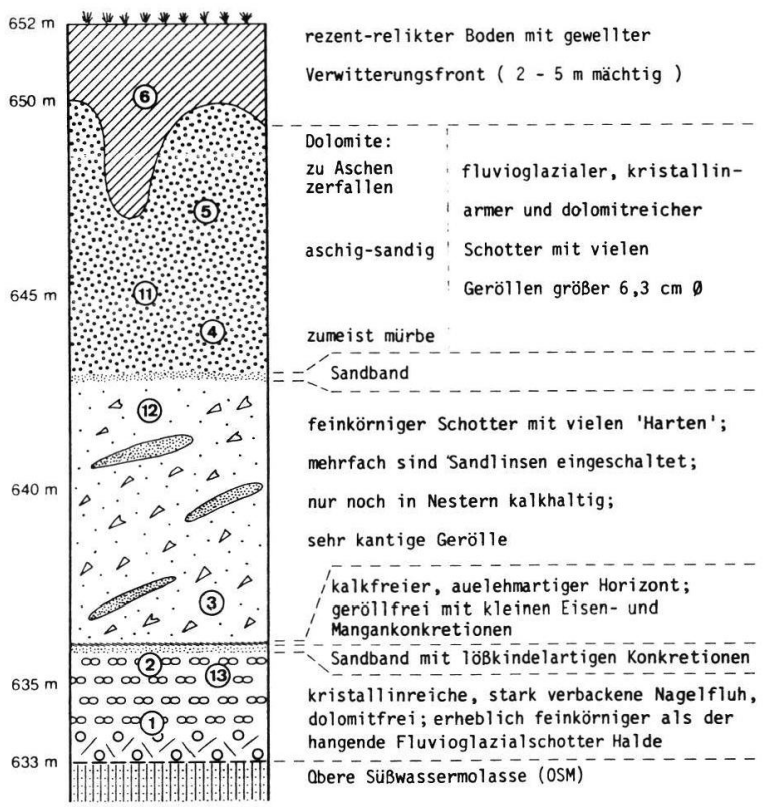

Schwermineralanalyse

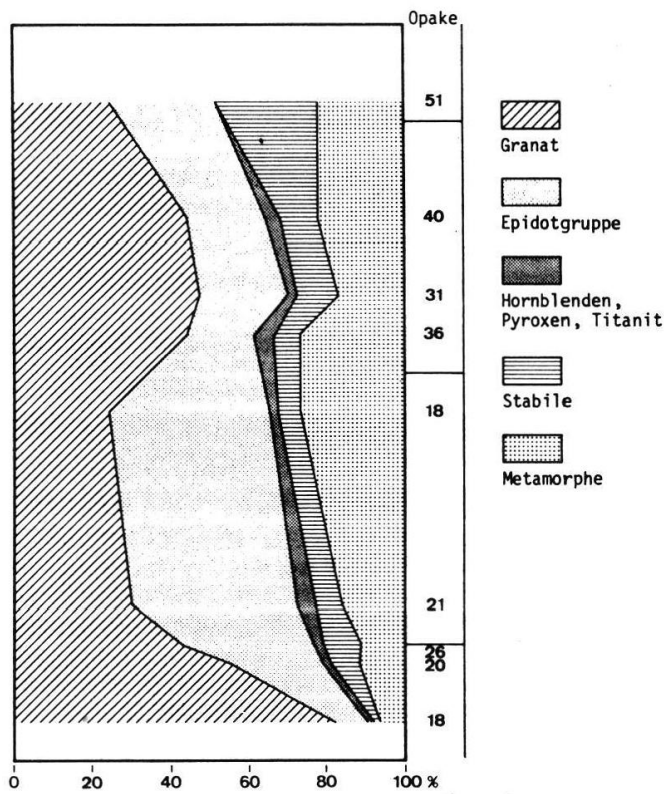

Abb. 2: Die Schichtenabfolge in der Kiesgrube am Schützenhaus von Daxberg.

Die Zahlen in den Kreisen markieren Probenentnahmestelle und Probennummer.

Im Schwermineraldiagramm kennzeichnen die Prozentzahlen der Opaken gleichzeitig die Entnahmehöhe der Proben.

Zurundung kommt vielfach über das Stadium kantig nicht hinaus.

Dies und die unten folgenden Schwermineralanalysen erlauben, den mittleren Schotter als eine Periglazialfazies anzusprechen, in der einerseits schon verwittertes Material aus Fließerden (Böden der vorhergegangenen Interglaziale) aufgearbeitet worden ist, in die aber auch frisches, kalkhaltiges Material (z. B. aus der Adelegg) eingearbeitet wurde. Nach Ablagerung dieser und vor Ablagerung des hangenden Schotters muß eine weitere Verwitterungsphase stattgefunden haben, denn anders sind die vorhandenen Kieselskelette nicht zu erklären ${ }^{8}$ ). Für ,in situ' Verwitterungsprozesse in diesem mittleren Schotter sprechen auch lößkindelartige Kalkanreicherungen in einem Sandband unter dem Schotter. Allerdings haben diese Prozesse nicht die Intensität erreicht, die erlauben würde, für sie ein eindeutig interglaziales Alter zu postulieren.

Über die Qualität der Verwitterungsprozesse sind nur schwer Aussagen möglich, denn das Sediment be-

8) Kieselskelette verwitterter Kalke zerbrechen bei solifluidalem Transport. Bei der vorgefundenen Menge kann auch nicht mit gefrorenen Kieselskeletten, die so den solifluidalen und fluvialen Transport überstanden hätten, argumentiert werden. stand ja schon zur Zeit seiner Ablagerung zumindest teilweise aus solifluidal umgelagertem und fluvial transportiertem Verwitterungsmaterial. Wieviel Sediment wie stark in situ subaerisch verwittert sein dürfte, ist kaum zu entscheiden. Der relativ geringe Tonanteil (Tab. 3) weist auf keine allzu intensiven Bodenbildungsprozesse etwa interglazialen Charakters hin ${ }^{9}$ ).

Die unteren $3 \mathrm{~m}$ der Kiesgrube werden von einer nahezu dolomitfreien, kristallinreichen, sehr stark verbackenen Nagelfluh eingenommen. Dieser Schotter wurde von SINN (1972) und JERZ (1978) als eine Ablagerung fluviatil-periglazialer Adelegg-Flüsse gedeutet, eine Interpretation, die nach Kenntnis einiger kristallinreicher Tertiärschottervorkommen der Adelegg sehr einleuchtend ist.

\subsubsection{Schwermineralanalysen}

Aus der gesamten Sedimentfolge wurden 9 Proben für Schwermineralanalysen gewonnen. Probe 6 wurde

9) Oder aber das vorgefundene Sediment stellt nur den untersten Teil eines gekappten Verwitterungsprofils $\left(B_{v}\right.$ oder $\mathrm{C}_{\mathrm{v}}$-Horizont) dar. Dann müßte allerdings eine erhebliche Zeitspanne zwischen Ablagerung des mittleren Sediments, der Verwitterung und der Akkumulation des hangenden Schotters verstrichen sein. 
Tab. 2: Schwermineralanalysen aus der Kiesgrube am Schützenhaus von Daxberg

\begin{tabular}{|c|c|c|c|c|c|c|c|c|c|c|c|c|c|c|c|}
\hline \multirow[b]{2}{*}{$\begin{array}{c}\text { Proben- } \\
\text { nr.: }\end{array}$} & \multirow[b]{2}{*}{$\begin{array}{l}\text { 藏 } \\
\text { 岕 }\end{array}$} & \multirow{2}{*}{ 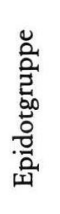 } & \multirow{2}{*}{ 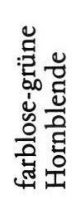 } & \multirow[b]{2}{*}{ 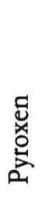 } & \multirow[b]{2}{*}{ 䔍 } & \multirow[b]{2}{*}{ 趨 } & \multicolumn{3}{|c|}{ Stabile } & \multicolumn{6}{|c|}{ Metamorphe } \\
\hline & & & & & & & 恚 & $\begin{array}{l}\text { 菩 } \\
\text { 品 } \\
\text { ص) }\end{array}$ & 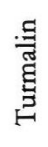 & 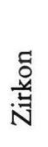 & 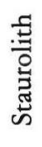 & 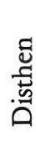 & 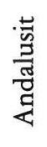 & 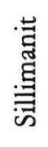 & $\begin{array}{l}\text { y. } \\
\text { مै }\end{array}$ \\
\hline 6 & 25 & 26 & 0 & 0 & 0 & 13 & 1 & 0 & 1 & 11 & 17 & 4 & 1 & 0 & 51 \\
\hline 5 & 44 & 20 & 3 & 0 & 1 & 6 & 1 & 0 & 3 & 0 & 14 & 4 & 4 & 0 & 40 \\
\hline 11 & 47 & 23 & 1 & 1 & 0 & 3 & 3 & 1 & 1 & 3 & 16 & 1 & 0 & 0 & 31 \\
\hline 4 & 43 & 18 & 5 & 0 & 0 & 2 & 1 & 0 & 3 & 1 & 22 & 4 & 1 & 0 & 36 \\
\hline 12 & 24 & 41 & 2 & 0 & 1 & 3 & 1 & 0 & 1 & 0 & 18 & 6 & 3 & 0 & 18 \\
\hline 3 & 30 & 42 & 4 & 0 & 1 & 1 & 1 & 0 & 3 & 2 & 11 & 2 & 2 & 1 & 21 \\
\hline 2 & 43 & 34 & 7 & 0 & 0 & 2 & 1 & 0 & 1 & 1 & 7 & 2 & 2 & 0 & 26 \\
\hline 13 & 55 & 24 & 2 & 0 & 0 & 3 & 1 & 0 & 1 & 2 & 9 & 2 & 1 & 0 & 20 \\
\hline 1 & 82 & 8 & 2 & 0 & 0 & 0 & 0 & 0 & 0 & 1 & 5 & 2 & 0 & 0 & 18 \\
\hline
\end{tabular}

zu Vergleichszwecken dem rezent-relikten Boden entnommen, Proben 4, 5 und 11 dem hangenden Fluvioglazialschotter, 3 und 12 dem mittleren Schichtpaket, 1, 2 und 13 der liegenden Nagelfluh (vgl. Abb. 2). Die vorliegenden Ergebnisse der Analysen (Abb. 2, Tab. 2) lassen eine Vierteilung des Profils in die drei verschiedenen Schotterfazies sowie in den hangenden Boden zu.

Die Proben aus der liegenden Nagelfluh $(1,2,13)$ zeigen durch das nicht verwitterungsbedingte Nachlassen des Granatanteils auf den Rückgang der aufgearbeiteten und dem Sediment einverleibten Molassekomponente hin. Die deutlich geringeren Granatgehalte in den Proben 3 und 12 aus dem mittleren Sediment dürften zumindest teilweise verwitterungsbedingt sein, da besonders in Probe 13 die Granate stark angewittert sind. Die Tatsache, daß die verwitterungsanfälligen Hornblenden noch vorhanden sind, macht die Interpretation des Milieus, in welchem das Sediment abgelagert wurde bzw. verwittert ist, nicht einfacher.

Das einheitlichste Spektrum weist der hangende fluvioglaziale Schotter auf (Proben 4, 5, 11) und da sich der Boden oberhalb (Probe 6) eindeutig auf diesem Schotter entwickelt hat, ist die Differenz im Schwermineralspektrum auf Verwitterungsprozesse zurückzuführen. Im Boden tritt der Granatanteil stark zurück, Hornblenden fehlen, die Stabilen sind dagegen häufig und der Anteil der Opaken ist so hoch wie in keiner der anderen Proben. Für die
Bodenbildungs- und Verwitterungsprozesse auf dem hangenden Schotter standen mehrere Interglaziale und das Holozän zur Verfügung, dem mittleren Schichtpaket wären für eine in situ Entkalkung weit geringere Zeitspannen zur Verfügung gestanden.

\subsubsection{Bewertung}

Schotter-, Schwermineral- und Korngrößenanalysen (Tab. 3) legen nahe, die Sedimentfolge am Schützenhaus von Daxberg dreizuteilen, wobei auf dem hangenden Schotter noch ein mächtiger Bodenhorizont ausgebildet ist.

Die liegende Nagelfluh, die primär nahezu dolomitfrei war, kann nach den Ergebnissen von SINN (1972) und JERZ (1978) auf umgelagerte Gerölle aus dem Adelegg-Schuttfächer zurückgeführt werden. In diesem Sediment ist fluvioglazialer Einfluß aus den nördlichen Kalkalpen nicht nachzuweisen, denn die dafür charakteristischen Dolomite fehlen nahezu vollständig.

Diese grobe und feinmaterialarme Nagelfluh endet unterhalb eines fast den ganzen Aufschluß durchziehenden Sandbandes, das von einem kalkfreien, sehr feinkörnigen, auelehmartigen Horizont nach oben hin abgeschlossen wird. Dieser Horizont läßt an eine nachlassende Schüttung groben Materials und/oder an ein Nachlassen der Schleppkraft der periglazialen Flüsse denken, sodaß zuerst noch Sand, dann nur noch Schluff und Ton abgelagert werden konnte. 
Tab. 3: Korngrößenanalysen aus der Kiesgrube am Schützenhaus von Daxberg, vom Hochfirst und Stoffersberg (Gerölle $>2,0 \mathrm{~cm} \varnothing$ von Hand abgesiebt und petrographisch analysiert, vgl. Tabelle 1)

$\begin{array}{cccc} & \text { Daxberg } & \text { Hochfirst } & \text { Stoffersberg } \\ \text { hangende Fazies } & \text { mittlere Fazies } & \text { liegende Fazies } & \end{array}$

\begin{tabular}{|c|c|c|c|c|c|}
\hline Mittelkies: & $19,0 \%$ & $41,7 \%$ & $68,3 \%$ & $21,3 \%$ & $33,7 \%$ \\
\hline \multirow[t]{2}{*}{ Feinkies: } & $7,7 \%$ & $19,2 \%$ & $8,6 \%$ & $8,1 \%$ & $11,0 \%$ \\
\hline & $26,7 \%$ & $60,9 \%$ & $76,9 \%$ & $29,4 \%$ & $44,7 \%$ \\
\hline \multirow{3}{*}{$\begin{array}{l}\text { Grobsand: } \\
\text { Mittelsand: } \\
\text { Feinsand: }\end{array}$} & $4,0 \%$ & $8,2 \%$ & $1,2 \%$ & $2,4 \%$ & $3,4 \%$ \\
\hline & $21,4 \%$ & $12,9 \%$ & $11,2 \%$ & $8,3 \%$ & $5,8 \%$ \\
\hline & $17,9 \%$ & $12,4 \%$ & $7,3 \%$ & $18,5 \%$ & $12,9 \%$ \\
\hline Sand & $43,3 \%$ & $33,5 \%$ & $19,7 \%$ & $29,2 \%$ & $22,1 \%$ \\
\hline \multirow{3}{*}{$\begin{array}{l}\text { Grobschluff: } \\
\text { Mittelschluff: } \\
\text { Feinschluff: }\end{array}$} & $18,5 \%$ & $2,0 \%$ & $1,4 \%$ & $25,3 \%$ & $19,5 \%$ \\
\hline & $6,0 \%$ & $1,1 \%$ & $1,0 \%$ & $13,2 \%$ & $11,1 \%$ \\
\hline & $1,6 \%$ & $0,8 \%$ & $0,7 \%$ & $1,1 \%$ & $1,3 \%$ \\
\hline Schluff & $26,1 \%$ & $3,9 \%$ & $3,1 \%$ & $39,6 \%$ & $31,9 \%$ \\
\hline Ton: & $3,9 \%$ & $1,7 \%$ & $0,3 \%$ & $1,8 \%$ & $1,3 \%$ \\
\hline
\end{tabular}

Auf ein Zusammenwirken von solifluidalen und fluviatil-periglazialen Prozessen geht die Akkumulation des mittleren Sediments zurück. Schrägschüttung und Kreuzschichtung weisen auf die Dominanz der fluvialen Dynamik hin. Postsedimentäre Entkalkung muß zumindest teilweise stattgefunden haben. Ein über die gesamte Aufschlußbreite ziehendes Sandbzw. toniges Band am Top der Ablagerung zeigt ein Erlahmen der Schleppkraft an.

Die Ansprache des hangenden Schotters als fluvioglaziales Sediment bereitet keinerlei Schwierigkeiten. Nach den Ergebnissen von LÖSCHER (1976) und LÖSCHER \& SINN (1983) ist das Alter höchstwahrscheinlich älter als viertletzteiszeitlich (d. h. älter als Günz im klassischen Sinne).

\section{Zur Genese und Stratigraphie der ältesten Schotter auf der südlichen Iller-Lechplatte}

Die höchstgelegenen Sedimente wie der Hochfirstschotter, der Plattenberg-Arlesrieder Schotter oder der Stoffersbergschotter haben im Schotterspektrum große Ähnlichkeit mit den jüngeren eindeutig fluvioglazialen Schottern. Sie unterscheiden sich aber grundlegend von periglazial-fluviatilen Ablagerungen, gleich ob diese als Restschotter oder restschotterartige Sedimente (mittlerer Bereich der Kiesgrube Daxberg) oder als umgelagerte Tertiärschotter (liegender Bereich der Kiesgrube Daxberg) vorliegen. Nach der petrographischen Zusammensetzung war das Liefergebiet der Ur-Iller, welche den Hochfirstschotter ablagerte, identisch mit dem Einzugsgebiet des Gletschers, dessen umgelagerte Sedimente das Hangende der Kiesgrube Daxberg bildet.

Da der Hochfirstschotter aber erheblich feinere Gerölle infolge eines längeren fluvialen Transportweges führt als der fluvioglaziale Schotter von Daxberg, ist eine zugehörende Eisrandlage weit südlich der bisher bekannten zu vermuten. Durch ihr Geröllspektrum sind Hochfirst- wie Stoffersbergschotter genetisch mit den jüngeren fluvioglazialen Sedimenten gleichzusetzen, allerdings ohne nachweisbare Moränenverbindung. 
Die ältesten Schotter des extramoränalen Bereichs zwischen Iller und Lech stammen alle aus dem Pleistozän, wobei tertiäre Konglomerate aus der OSM wie nördlich des Auerbergs (RÖGNER 1979) oder bei Schellenberg (JERZ 1983) natürlich nicht mit den pleistozänen Ablagerungen verwechselt werden dürfen. Im Bereich der südwestlichen Iller-Lechplatte ist der Gehalt an Dolomiten das wichtigste Unterscheidungskriterium; denn hier bleiben die Dolomitgehalte der tertiären Konglomeratlagen meist weit unter $10 \%$, zum Teil sind sie dolomitfrei (SCHIEMENZ 1960), bei den quartären Schottern liegen sie meist über $30 \%$ (dazu auch SinN 1972: 51 und 52; Anm. zu Tab. 5 und 6).

Auf der südöstlichen Iller-Lechplatte bieten die, gelben Kalke', die in den quartären Ablagerungen $30 \%$ nicht erreichen, in den tertiären Nagelfluhen des Weichberges aber $85 \%$ (RÖGNER 1979) ausmachen, das einfachste Unterscheidungskriterium.

Die Schotterreste auf dem Hochfirst und Stoffersberg wurden während der ältestpleistozänen Biberkaltzeiten akkumuliert. Ihr Habitus unterscheidet sich von den biberkaltzeitlichen Schottern der Typenregion westlich Augsburg (SCHAEFER 1957) vor allem durch die Tatsache, daß in ihnen noch Kalke und sogar Dolomite vorhanden sind, während die ältestpleistozänen Schotter am Staufersberg (Staufenberg) vollständig entkalkt sind.

Der hangende Schotter in der Kiesgrube am Schützenhaus von Daxberg wird in die jüngere Donaueiszeit eingeordnet, das Alter der mittleren und liegenden Sedimente ist ebenfalls donaueiszeitlich, gegebenenfalls aber aus einer älteren Phase.

\section{Schluß}

Die ältesten Schotter der südlichen Iller-Lechplatte auf dem Hochfirst und dem Stoffersberg haben große Ähnlichkeit mit den jüngeren, eindeutig fluvioglazialen Ablagerungen. Deshalb müssen zur Zeit der jeweiligen Sedimentation in etwa identische Ablagerungs- und Herkunftsbedingungen geherrscht haben. Die Tatsache, daß sie sich in ihrer Petrographie eindeutig von den älteren, tertiären Nagelfluhen aus der OSM, aber auch von kaltzeitlichen, periglazial-fluviatilen Ablagerungen unterscheiden, muß zu dem Schluß führen, daß ihre Entstehung mit Vereisungen, die zumindest bis in die Nähe des Nordalpenrandes oder darüber hinaus vorgestoßen waren, die aber die Jungendmoränenwälle nicht erreicht haben, zurückgeführt werden. Da Klimaverschlechterungen, die zu Vereisungen geführt haben, erst im Pleistozän aufgetreten sind, ist ein pleistozänes Alter sehr wahrscheinlich und man kann von Bibereiszeiten sprechen.

\section{Dank}

Herzlichen Dank schulde ich Prof. Dr. Hans Graul, meinem Lehrmeister und Doktorvater, mit dem ich das Alter der ,ältesten” Schotter viele Stunden diskutieren konnte. Ihm sei dieser Aufsatz nachträglich zu seinem 75 . Geburtstag gewidmet.

Die Schwermineralanalysen wurden dankenswerterweise und großzügig wie immer von Priv. Doz. Dr. W. Tillmanns (Köln) durchgeführt.

Finanzielle Unterstützung erhielt ich im Verlaufe der Jahre von der Deutschen Forschungsgemeinschaft, der Konrad Adenauer-Stiftung und der Universität-Gesamthochschule Paderborn.

\section{Schriftenverzeichnis}

EBERL, B. (1930): Die Eiszeitenfolge im nördlichen Alpenvorlande. - VIII + 427 S., 19 Abb., 2 Taf., 1 Kte.; Augsburg (Filzer).

Graul, H. (1962): Eine Revision des pleistozänen Stratigraphie des schwäbischen Alpenvorlandes (mit einem bodenkundlichen Beitrag von K. BRUNNACKER). Petermanns Geographische Mitteilungen, 106: 253-271, 8 Abb.; Gotha.

—, Schaefer, I. \& Weidenbach, F. (1951): Quartärgeologische Exkursion durch die Riß-Lechplatte. - Geologica Bavarica, 6: 91-117, 9 Abb.; München.

JERZ, H. (1978): Quartär. - In: Erläuterungen zum Blatt Nr. 7927 Amendingen der Geologischen Karte von Bayern (1:25 000) von JERZ \& WAGNER: 41—65, 5 Abb., 2 Tab.; München.

- (1983): Halt 10 der Exkursion J am 9. 4. 1983. In: Quartär in der Umgebung von Obergünzburg. Jbr. Mitt. oberrhein. geol. Verein, N.F., 65: 130; Stuttgart (Schweizerbart).

- \& W. Grottenthaler (1981): Glazialer und fluvioglazialer Bereich. - In: Erläuterungen zur Geologischen Karte von Bayern (1:500000), 3. Aufl.: 135-141, 4 Tab.; München.

—, Stephan, W., Streit, R. \& Weinig, H. (1975): Zur Geologie des Iller-Mindel-Gebietes. - Geologica Bavarica, 74: 99-130, 2 Beil.; München.

- \& WAGNER, R. (1978): Geologische Karte von Bayern $(1: 25000)$, Blatt Nr. 7927 Amendingen: 131 S., 20 Abb., 7 Tab., 4 Beil.; München.

LOSCHER, M. (1976): Die präwürmzeitlichen Schotterablagerungen in der nördlichen Iller-Lechplatte. - Heidelberger geogr. Arb., 45: IX + 157 S., 26 Abb., 4 Tab., 8 Taf., 4 Ktn., 17 Beil.; Heidelberg.

— \& SinN, P. (1983): Ein Beitrag zur Quartärstratigraphie und Paläogeographie des „Eisenburger Schotterfeldes” nördlich Memmingen. - Mitt. Geograph. Ges. München, 68: 67-88, $6 \mathrm{Abb}$.; München. 
MADER, M. (1976): Schichtenfolge und Erdgeschichte im Bereich des Schussenlobus des pleistozänen Rhein-Vorlandgletschers. - [Unveröff.] Diss. Univ. Tübingen: 169 S.; Tübingen.

Penck, A. \& BRÜCKNer, E. (1901-09): Die Alpen im Eiszeitalter. - 1199 S., 156 Abb., 30 Taf., 19 Ktn.; Leipzig (Tauchnitz).

RÖGNER, K. J. (1979): Die glaziale und fluvioglaziale Dynamik im östlichen Lechgletschervorland. - Ein Beitrag zur präwürmzeitlichen Pleistozänstratigraphie. - Heidelberger geogr. Arb., 49: 67-138, 12 Fig., 5 Abb., 5 Ktn., 2 Prof., 3 Beil.; Heidelberg.

SCHIEMENZ, S. (1960): Fazies und Paläogeographie der subalpinen Molasse zwischen Bodensee und Isar. Beih. Geol. Jb., 38: 119 S., 23 Abb., 17 Tab., 6 Taf.; Hannover.

SCHAEFER, I. (1951): Quartärgeologische Exkursion durch die Riß-Lechplatte. - In: Graul, Schaefer \& WeidenBACH, 1951: 98-114, 4 Abb.; München.

- (1953): Die Donaueiszeitlichen Ablagerungen an Lech und Wertach. - Geologica Bavarica, 19: 13-65, 15 Abb.; München.

- (1957): Erläuterungen zur Geologischen Karte von Augsburg und Umgebung (1:50 000). - 92 S., 4 Abb., 2 Beil.; München.

- (1965): The sucession of fluvioglacial deposits in the Northern Alpine Foreland. - Proceedings of the VII. Congress/INQA, 14: 9-14; Boulder-Denver, Colorado.

\section{Kartenverzeichnis}

JERZ, H. (1978): Geologische Karte von Bayern (1:25 000), Blatt Nr. 7929 Amendingen; München (Geologisches Landesamt).

SCHAEFER, I. (1957): Geologische Karte von Augsburg und Umgebung (1:50000); München (Geologisches Landesamt).

ScholZ, H. (1983): Geologische Übersichtskarte der Bundesrepublik Deutschland $(1: 200000)$, Blatt Nr. CC 8726 Kempten; Hannover (Bundesanstalt für Geowissenschaften und Rohstoffe). Die wissenschaftliche Bearbeitung des Quartärs erfolgte durch H. ScHOLZ.

Streit, R., Weinig, H., Jerz, H. \& Stephan, W. (1975): Geologische Übersichtskarte des Iller-Mindel-Gebietes (1:100 000) mit Gewinnungsstellen für Lockergesteine; München (Geologisches Landesamt).

Topographische Karte 1:25000; Blatt Nr. 7927 Amendingen, Blatt Nr. 7930 Buchloe, Blatt Nr. 8028 Markt Rettenbach; München (Bayerisches Landesvermessungsamt). 
